\title{
Study on evaluation of MRI in Internal derangements of Knee Joint
}

\author{
Sharana Basappa G M
}

Professor and HOD, Department of Radiology, The Oxford Medical College \& Research Centre, Bangalore- 560011.

Email: sharanabasappa@gmail.com

Abstract Background: The knee is one of the most commonly involved joint in the external injuries. Internal derangement of knee (IDK) joint is a common cause of morbidity in the young, active individuals like athletes. The most widely used investigations are arthroscopy and Magnetic Resonance Imaging (MRI). Arthroscopy is considered as the gold standard for diagnosis of traumatic intra-articular lesions; however, it is an invasive procedure requiring hospitalization and anaesthesia and is associated with complications. Hence Magnetic Resonance Imaging (MRI) has now been accepted as the best imaging modality for non-invasive evaluation of knee injuries. This study has been done to know the pattern of distribution of different types and incidence of injuries in traumatic knee joint by MR imaging and to compare with arthroscopy or arthrotomy findings. To evaluate the pattern of distribution of ligamental and mensical injuries using Magnetic Resonance Imaging (MRI). To find the proportion of cases where the MRI findings agree with arthroscopy or arthrotomy findings. Materials and Methods: 69 Patients who undergo MR imaging of the knee on the advice of the referring doctor on his suspicion of internal derangement of the knee (IDK) in the department of Radiodiagnosis served as the subjects for this study. Arthroscopy was carried out in OT by orthopaedic surgeons in selected and available cases for diagnostic or therapeutic purposes. Findings of MRI of the Knee are correlated with the arthroscopic findings in patients with internal derangements of knee. Results- Qualitative data is described in terms of percentage from the 69 patients with suspected internal derangement of knee joint evaluated with MRI with assessment of $95 \%$ confidence interval and in selected cases proportion of agreement with the arthroscopy or arthrotomy is obtained. Conclusion- MRI is a noninvasive, useful, noninvasive, radiation free and reliable diagnostic tool for evaluating knee injury and it should be done in suspected menisci and ligamentous injury, to be posted for arthroscopy, thus preventing unwanted diagnostic arthroscopy.

Key Words: MRI, Arthroscopy, IDK.

Address for Correspondence:

Dr. Sharana Basappa G M, Professor and HOD, Department of Radiology, The Oxford Medical College \& Research

Centre, Bangalore- 560011.

Email:sharanabasappa@gmail.com

Received Date: 20/07/2019 Revised Date: 15/08/2019 Accepted Date: 22/09/2019

DOI: https://doi.org/10.26611/10131311

\begin{tabular}{|l|l|}
\hline \multicolumn{2}{|c|}{ Access this article online } \\
\hline Quick Response Code: & Website: \\
\hline & www.medpulse.in \\
\hline
\end{tabular}

\section{INTRODUCTION}

The knee is one of the most commonly involved joint in the external injuries. Internal derangement of knee joint is a common cause of morbidity in the young, active individuals like athletes. Early detection of the cartilage and ligament abnormalities is vital for early intervention to prevent further degeneration. The most widely used investigations are arthroscopy and MRI. Arthroscopy is considered as the gold standard for diagnosis of traumatic intra-articular knee lesions, however, it is an invasive procedure requiring hospitalization and anaesthesia and is associated with complications. Hence Magnetic Resonance Imaging (MRI) has now been accepted as the best imaging modality for non-invasive evaluation of knee injuries. When compared with other diagnostic methods, MRI has the advantage of demonstrating the cartilages, bones, soft tissues and ligaments directly, in detail and in different planes. With the availability of the specialized extremity coil, the knee has become the most frequently studied articulation on MRI. The purpose of this study was to find out the various types 
of traumatic lesions of the knee on MRI, to correlate the results with arthroscopy, and to establish the accuracy of MRI in detecting ligament and meniscus injury considering arthroscopy as gold standard. This study has been done to know the pattern of distribution of different types and incidence of injuries in traumatic knee joint by MR imaging and to compare with arthroscopy or arthrotomy findings in cases applicable.

\section{Internal Derangement of Knee - MRI Appearances} The Cruciate Ligaments: Anterior cruciate ligament: MRI signs - The direct signs include 1,2,3 Discontinuity of ACL fibers. ii) Abnormal contour of anterior cruciate ligament. The indirect signs include 1,4 i) Buckling of posterior cruciate ligament.ii)Anterior translation of tibia. iii) Overhanging of posterior horn of the lateral meniscus. iv) Deep lateral femoral sulcus exceeding $2 \mathrm{~mm}$ in depth v) Segond fracture of the lateral tibia. vi) Chip fracture of the posterior tibia vii) kissing bone contusion. Subacute phase is the best time for evaluation of tear. Proximal end may be displaced posterior to the PCL giving the appearance of a loose body. Chronic ACL tears are difficult to evaluate due to associated atrophy. The distal end of the ACL may be seen intact and attached to the PCL without visualization of the proximal end and is commonly reported as normal. Posterior cruciate ligament (PCL): MRI appearances include thickening of the middle portion of the ligament with hyperintensities on both T1- and T2- weighted images. Unlike acute ACL tears, continuity of the ligament is maintained in the PCL with acute trauma ${ }^{3,5}$. Chronic tears of the PCL are difficult to diagnose because the ligament will form a fibrous scar in the place of normal ligament which is of same signal intensity rest of the ligament. Secondary signs include ligament laxity or persistent hyperintensity ${ }^{3,6}$

The Collateral Ligaments: Medial collateral ligament (MCL) Grading of MCL tears; Grade I: Intact MCL fibers with hyperintenisty superficial to the MCL due to edema Grade II: Partial tear of MCL Grade III: Complete tear of the MCL fibers with associated edema . Lateral collateral ligament (LCL) LCL injuries are usually associated with other injuries $^{7}$. The common associations are injuries of the capsule, the biceps femoris, and the popliteus, less commonly also cruciate ligaments or the lateral tibial rim (Segond fracture) and usually as a part of posterolateral corner injury ${ }^{8,9}$. The MENISCI Compression and rotation with abnormal shearing forces of knee results in meniscal damage. Clinicaly patient may present with joint pain, giving way, clicking, locking of knee in fixed flexion and swelling.
MENISCAL Tears: Diagnostic Criteria: Two criteria for diagnosing a meniscal tear are commonly used ${ }^{10}$ : i) An hyperintense intrasubstance area extending to the articular surface, Or ii) Abnormal meniscal morphology. Grading of meniscal injury Grade 1: Intrameniscal hyperintensity not extending to the articular surface. Grade 2: Linear signal intensity that does not intersect the inferior or superior articular surface but is in contact with the capsular margin at the posterior aspect of the meniscus. Grade 3: Hyperintensity extending to the superior and/or inferior articular surface. Grade 1 and grade 2 lesions indicate mucinous or mucoid intrasubst-ance degenerative change and are usually encountered after 3 or 4 decade. Due to prominent vascularity in children and young adults misinterpretation as grade 1 or 2 tear is usual. ${ }^{4,11}$ Classification of tears: 1. Horizontal tears 2.Vertical tears 3. Complex tears. Discoid Meniscus, Meniscal Ossicles and Meniscal flounce are normal variants ${ }^{4,12}$. Osseous and Osteochondral Lesions: Segond and reverse Segond fractures, Osteochondral lesi-ons and Spontaneous osteonecrosis of the knee.

\section{MATERIALS AND METHODS}

A total number of 72 cases with IDK who underwent MR imaging of knee in the Radiology department, served as the subjects for this study. All patients are subjected to MR imaging and followed by arthroscopy in selected cases (wherever applicable. MRI i. T1 and PDFS weighted sequences in sagittal and coronal planes. ii. T2- weighted in axial, coronal and sagittal planes. MRI Data evaluated for: Joint effusion , Anterior Cruciate Ligament tear , Partial/Complete Posterior Cruciate Ligament tear, Partial/Complete Medial Collateral Ligament tear with grading, Lateral Collateral Ligament tear with grading, Medial Meniscal Tear with grading, Lateral Meniscal Tear with grading and Osseous /Osteochondral lesions. Arthroscopy/ Arthrotomy was carried out in OT by orthopedic surgeons in selected and available cases for diagnostic or therapeutic purposes. Statistical Methods: i]. Descriptive data analysis with assessment of $95 \%$ confidence interval Agreement of MRI with arthroscopy or arthrotomy in terms of percentage is analysed . Ethical Consideration: Study was conducted only after getting approval from Institutional Ethical Committee. Written informed consent was taken from all the patients presenting with internal derangement of knee undergoing MRI knee joint in our department the same was followed. And in selected cases MRI was followed by arthroscopy after taking proper written inform consent in the department of orthopedics. 


\section{RESULTS AND ANALYSIS}

Study Design: Qualitative data is described in terms of percentage from the 72 patients with suspected internal derangement of knee joint evaluated with MRI with assessment of $95 \%$ confidence interval and in selected cases proportion of agreement with the arthroscopy or arthrotomy is obtained.

\begin{tabular}{|c|c|c|c|}
\hline \multicolumn{2}{|c|}{ MRI FINDINGS } & \multicolumn{2}{|c|}{$\begin{array}{l}\text { POSITIVE } \\
\text { FINDINGS \% }\end{array}$} \\
\hline \multicolumn{2}{|c|}{ JOINT EFFUSION 53} & \multicolumn{2}{|c|}{76.82} \\
\hline ACL TEAR & 49 & \multicolumn{2}{|c|}{71.01} \\
\hline PCL TEAR & 7 & \multicolumn{2}{|c|}{10.15} \\
\hline MCL TEAR & 9 & \multicolumn{2}{|c|}{13.04} \\
\hline LCL TEAR & 10 & \multicolumn{2}{|c|}{14.49} \\
\hline MM TEAR & 19 & \multicolumn{2}{|c|}{27.54} \\
\hline LM TEAR & 11 & \multicolumn{2}{|c|}{15.94} \\
\hline \multicolumn{2}{|c|}{ OSSEOUS OR OSTEOCHONDRAL } & \multicolumn{2}{|c|}{13.05} \\
\hline \multicolumn{4}{|c|}{ Table 2: Arthroscopic findings } \\
\hline ARTHROSCOPIC & NUMBER & $\%$ & $95 \% \mathrm{Cl}$ \\
\hline FINDINGS & $(n=69$ & & \\
\hline AVAILABLE & 16 & 23.1 & 14.8- 34.4 \\
\hline NOT AVAILABLE & 53 & 76.9 & $65.6-85.1$ \\
\hline
\end{tabular}

Table 3: Agreement of MRI diagnosis with Arthroscopic findings

\begin{tabular}{lccr}
\hline MRI & NUMBER & $\%$ & \multicolumn{1}{c}{$95 \% \mathrm{Cl}$} \\
\hline AGREEMENT & $(\mathrm{N}=16$ & & \\
YES & 14 & 87.5 & $63.98-96.5$ \\
NO & 21 & 2.5 & $3.5-36.02$ \\
\hline
\end{tabular}




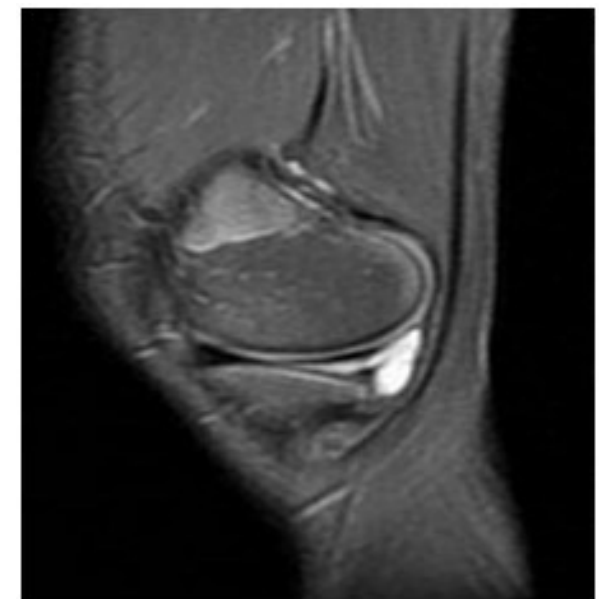

Figure 1

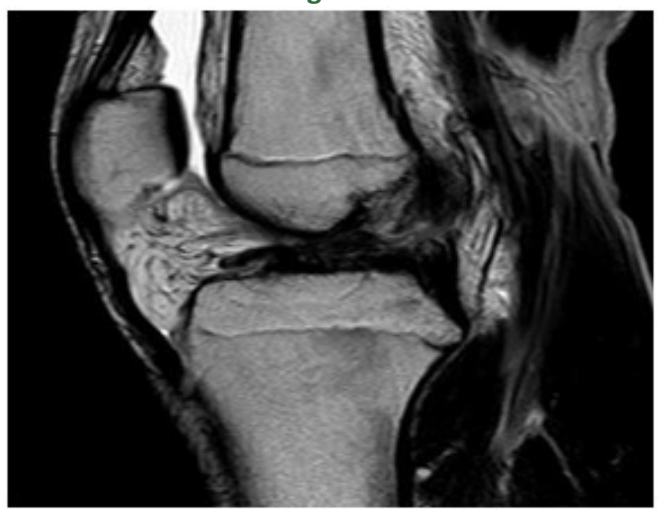

Figure 2

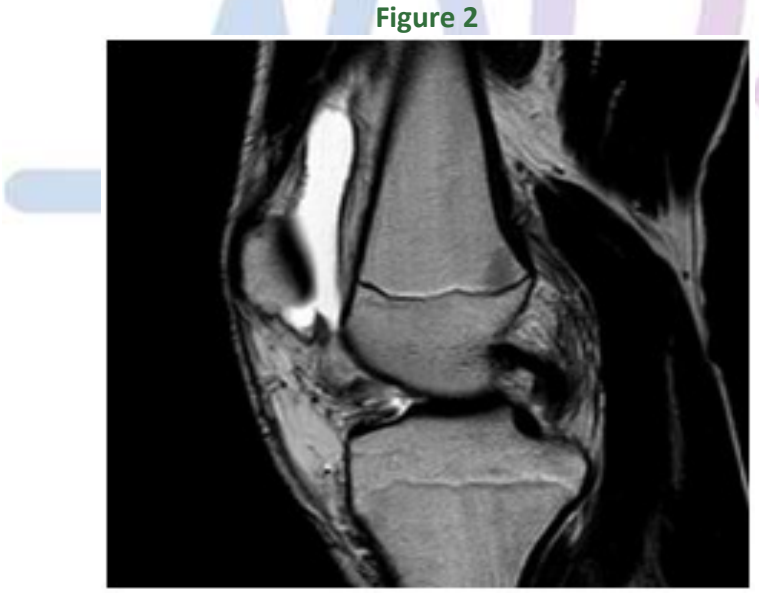

Figure 3

Figure 1: Sag T2 shows complete tear of ACL; Figure 2: Sag T2 showing buckled but intact PCL; Figure 3: Sag T2FS showing tear communicating with parameniscal cyst

\section{DISCUSSION}

Magnetic resonance imaging has now become the first line investigation for most of the knee lesions including pre and post operative evaluation. It allows complete evaluation of all the internal structures of the knee unlike other modalities like conventional radiography, arthrography, ultrasonography and computed tomography. Even with arthroscopy there is limitatations for detecting lesions such as peripheral meniscal tears, inferior surface tear. MRI has definite advantage in such cases. In our study joint effusions were the most common finding affecting 53 patients
(76.82\%). Among the ligamentous and meniscal injuries, ACL tear is most common, seen in 49 patients $(71.01 \%)$, to be followed by the Medial meniscal injuries seen in 19 patients $(27.54 \%)$ with grade 3 type injury being commonest. Cruciate ligaments: In our study ACL tear was found in 49 patients $(71.01 \%)$ among these $26(37.68 \%)$ were partial tears and 23 (33.33\%) were complete. Singh JP et al ${ }^{13}$ in their series of 173 patients, 78 patients (45.08\%) showed ACL tears, among these 52 (66.67\%), are partial, $16(20.51 \%)$ are complete. ACL tears are more common with partial tears being more 
common. Posterior cruciate ligament injuries were found to be relatively uncommon, in our study found in only 7 patients $(10.15 \%)$ and among that 6 tears were demonstrated as thickening of the ligament with abnormal signal intensity (partial tear) 1 was complete tear. Sonnin et al ${ }^{14}$ found the incidence of PCL tear to be 3 percent. Collateral ligaments: We have found in our study LCL tears $(14.49 \%)$ are commoner than the MCL tear (13.04\%). Grade 3 (5.75\%) MCL tear were more common in our study. Most of the collateral injuries were associated with other ligamental and meniscal injuries which is in concordance with the study of Mink JH etal. ${ }^{9}$ They observed on MRI and arthroscopy of 11 patients who had tear of LCL, 7 patients had tear of MCL, 4 patients had tear of lateral meniscus and 1 patient had tear of medial meniscus. Menisci lesions: In our study, MM tears ware found in $19(27.56 \%)$ with Grade I tear in $3(4.35 \%)$, Grade 2 tear in 1 (1.45\%)and Grade3 tear in $15(21.74 \%)$ and LM tear in 11(15.94\%) with Grade I tear in 4.34 (4.34) Grade 2 tear in $2(2.90 \%)$ and Grade 3 in 6 $(8.70 \%)$. Grade III tears were the more common in both the menisci; There is preponderance of MM tears over LM tears in our study which is well correlated with the study done by Singh JP et al, in a series of 173 cases of which they found 57 (38.23\%) patients showed MM tear and 28(29.41\%) patients showed LM tear. Out of 173 patients, Grade 3 tear of MM was seen in $57(32.95 \%)$ patients, Grade 2 in $16(9.25 \%)$ patients and Grade 1 in 20(11.56\%). In LM, Grade 3 tears were seen in $28(16.18 \%)$ patients, Gr 2 in 12 (6.94\%) patients and Gr I in 14 (8.1\%) patient. Parameniscsal cysts were In the 2 cases of the popliteal cysts, its location, relation to the joint space and its communication with joint space ware clearly demonstrated on sagittal T2 weighted images. These findings were correlated with findings described by Thomas H. Berquist. ${ }^{15}$ Osseous and Osteochondral injuries. In our study Osseous/Osteochondral lesions were seen in 9 patients. Most of these were bony contusions involving the femoral and tibial condyles. Osteochondral lesions are seen in nine patients. There is also fracture of medial tibial condyle in posterolateral aspect which is displaced antero-medially. Two case of Segond fracture of the lateral tibial condyle with bony contusion of the lateral femoral condyle was also seen. These findings were correlated with findings described by Thomas H.Berquist. ${ }^{15}$ In our study we have seen a single case of spontaneous osteonecrosis of medial femoral Condyle. It appeared as a focus of hyperintense signal in the antero-medial part of medial femoral condyle with a thin rim of low signal suggestive of sclerosis and associated marrow edema involving medial femoral condyle. These findings were correlated with findings described by Thomas H. Berquist. The finding of haemarthrosis and lipohemoarthrosis were seen in two cases. These findings were correlated with findings described by Thomas H. Berquist49. In our study, a agreement of MRI findings with arthroscopic findings was performed in 16patients $(23.1 \%)$. Among which in 14 patients $(87.5 \%)$ MRI findings are well correlated with arthroscopic findings with 95\% Confidence interval; 95\% CI (63.98- 96.5\%). ${ }^{16},{ }^{17}$ Combined injuries: In our study, we found 32 cases of combined injuries and 33 cases of isolated injuries. The predominant pattern was found to be a combination of anterior Cruciate ligament with lateral meniscus (9 patients). It is followed by a combination of ACL with LCL ( 8 patients) and ACL with MCL (8 patients) in equal numbers. Combination of ACL and medial meniscus tear was found in 4 patients.

\section{CONCLUSIONS}

The following patterns of knee injuries were seen: Most common injury was found to be ACL tear in which complete tears were more common than partial tears. Posterior cruciate ligament tears were less common. Among the Meniscal injuries medial Meniscal tears outnumbered lateral meniscus and grade 3 tears are more common in both. Lateral collateral ligament tears outnumbered medial ligament tears and grade 3 tears are more common in collateral ligaments. Osseous/Osteochondral lesions were seen in 52 patients $(46.8 \%)$. Most of these were bony contusions involving the femoral and tibial condyles. Good statistical co-relation was seen between MRI findings and Arthroscopic findings. (87.5\% cases showed excellent co-relation). Other abnormalities like discoid meniscus (1), hemarthrosis (2), joint effusions (89), and other myotendinous tears were also seen and characterized with the help of magnetic resonance imaging MRI is a noninvasive, useful, noninvasive, radiation free and reliable diagnostic tool for evaluating knee injury and it should be done in suspected menisci and ligamentous injury, prior to arthroscop , thus preventing unwanted diagnostic arthroscopy. It can accurately detect, localize and characterize various internal derangements of the knee joint with excellent soft tissue delineation and help in arriving at a correct anatomical diagnosis thereby helping further management of the patient.

\section{REFERENCES}

1. Crema MD, Hunter DJ, Roemer FW, et al. The relationship between prevalent medial meniscal intrasubstance signal changes and incident medial meniscal tears in wo-men over a 1 year period assessed with 3.0

2. Marc A. Camacho. The Double Posterior Cruciate Ligament Sign. Radiology 2004; 233:503- 504.

3. Sonin AH, Fitzgerald SW, Friedman H: MR imaging of Posterior cruciate ligament; Normal, Abnormal and associated injury pattern. Radiographics 1995; 15: $551-561$ 
4. Ryu KN, Kim IS, Kim EJ, et al. MR imaging of tears of discoid lateral menisci. AJR 1998; 171:963-967

5. Grover JS, Bassett LW, Gross ML. Posterior cruciate ligament: MR imaging. Radiology 1990;174:527530.

6. Akisue T, Kurosaka M, Yoshiya S. Evaluation of healing of the injured posterior Cruciate Ligament: analysis of Instability and Magnetic Resonance Imaging. Arthroscopy 2001;17(3): 264-269.

7. Miller TT, Gladden P, Staron RB, Henry JH, Feldman F: Posterolateral Stabilizers of the knee : anatomy and injuries assessed with MR imaging. AJR Am J Roentgenol 1997;169(6):1641-1647.

8. Edwin $\mathrm{H}$ G Oei, Abida Z Ginai, M G Myriam Hunink. MRI for Traumatic Knee Injury: A Review. Semin Ultrasound CT MRI 2007;28:141-157.

9. Mink JH: The Cruciate and Collateral ligaments, in Mink JH, Reicher MA, Crues JV III, (eds) : MRI of the Knee (2nd ed ). New York, Raven, 1993, pp 141188. Edwin H G Oei, Jeroen J Nikken, Antonia C M Verstijnen, Abida Z Ginai, M G Myriam Hunink.MR Imaging of the Menisci and Cruciate Ligaments: A Syst-ematic Review. Radiology2003;226:837- 848 .
10. Samoto N, Kozuma M, Tokuhisa T, et al. Diagnosis of Discoid Lateral Meniscus of the Knee on MR imaging. Magn Reson Imaging 2002; 20(1):59-64.

11. Michael G. Fox, MR Imaging of the Meniscus: Review, Current Trends, and Clinical Implications; radiolclin N Am 2007; 45:1033-1053.

12. Singh JP, Garg L, Shrimali R, Setia V, Gupta V. MR Imaging of knee with arthroscopic correlation in twisting injuries. IJRI 2004;14: 33-40.

13. Andrew H Sonin,Fitzerald SW,Friedman $\mathrm{H}$,et al . PCL injury : MRI diagnosis and pattern of injury .Radiology 1994 167:121-12

14. Thomas H. Berquist. Osseous and Myotendinous Injuries About the Knee. Radiolclin N Am2007;45: 955-968.

15. Bernard Rosner, Fundamentals of Biostatistics. In: Bernard Rosner, editor. Fundamental of Biostatistics. 5thed. Duxbury: Blackwell, 2000; 80-240.

16. M. Venkataswamy Reddy, Statistics for Mental Health Care Research. In: Subba Rao, S Jingar editors, NIMHANS, INDIA 2002.

Source of Support: None Declared Conflict of Interest: None Declared 